\title{
Symmetric duality for nondifferentiable multiobjective fractional variational problems involving cones
}

\author{
Young Min Kang ${ }^{1}$, Do Sang Kim ${ }^{1 *}$ and Moon Hee Kim²
}

\section{${ }^{*}$ Correspondence:}

dskim@pknu.ac.kr

${ }^{1}$ Department of Applied

Mathematics, Pukyong National

University, Busan, 608-737, Republic of Korea

Full list of author information is available at the end of the article

\begin{abstract}
We introduce a pair of symmetric dual problems for nondifferentiable multiobjective fractional variational problems with cone constraints over arbitrary cones. On the basis of weak efficiency, we obtain symmetric duality relations for Mond-Weir-type problems under invexity and pseudoinvexity assumptions. Our symmetric duality results extend and improve some known results in Mishra et al. (J. Math. Anal. Appl. 333:1093-1110, 2007) to the cone constraints.
\end{abstract}

MSC: $90 \mathrm{C} 29 ; 90 \mathrm{C} 32 ; 90 \mathrm{C} 26$

Keywords: symmetric duality; fractional variational problems; cone constraints; support functions

\section{Introduction}

The notion of symmetric duality in nonlinear programming, in which the dual of the dual is the primal, was first introduced by Dorn [1]. Dantzig et al. [2] discussed symmetric dual programs and established a symmetric duality under the convexity-concavity assumption. Mond and Hanson [3] first formulated a pair of symmetric dual variational problems by providing continuous analogue of the symmetric dual pair of Dantzig et al. [2] and proved the usual duality theorems under the convexity-concavity assumption. Suneja et al. [4] formulated a pair of Wolfe-type multiobjective symmetric dual programs over arbitrary cones, in which the objective function is optimized with respect to an arbitrary closed convex cone by assuming the functions involved to be cone-convex. Later on, Khurana [5] formulated a pair of Mond-Weir-type multiobjective symmetric dual programs over arbitrary cones and derived the symmetric duality theorems involving cone-pseudoinvex and strongly cone-pseudoinvex functions. Recently, Kim and Kim [6] extended the results of Suneja et al. [4] and Khurana [5] to nondifferentiable multiobjective symmetric dual programs for weak efficiency involving cone-invex and cone-pseudoinvex functions. Very recently, Ahmad et al. [7] extended the results of Suneja et al. [4] and Khurana [5] to a pair of multiobjective mixed symmetric dual programs over arbitrary cones. On the other hand, Chandra et al. [8] first introduced a symmetric duality in nonlinear fractional programming. Mond and Schechter [9] studied nondifferentiable symmetric duality, in which the objective function contains a support function. Following Mond and Schechter [9], Yang et al. [10] presented a pair of symmetric dual nonlinear fractional programming problems and established duality theorems under pseudo-convexity/pseudo-concavity as-

(c) 2013 Kang et al.; licensee Springer. This is an Open Access article distributed under the terms of the Creative Commons Attribution License (http://creativecommons.org/licenses/by/2.0), which permits unrestricted use, distribution, and reproduction in any medium, provided the original work is properly cited. 
sumptions on the kernel function. Further, Gulati et al. [11] generalized these results to static and continuous nonlinear fractional programming. For the multiobjective case of static nonlinear fractional program, symmetric duality was established under convexity assumptions. Subsequently, Gulati et al. [12] and Kim and Lee [13] gave two pairs of multiobjective symmetric dual variational programs, in which duality results were obtained under pseudoconvexity-pseudoconcavity and invexity assumptions, respectively. Chen [14] and Kim et al. [15] discussed duality results for multiobjective symmetric fractional variational programs involving invex functions. Recently, Mishra et al. [16] gave a symmetric dual pair for a class of nondifferentiable multiobjective fractional variational problems. Weak, strong, converse and self-duality relations were established under certain invexity assumptions. Recently, Ahmad et al. [17] formulated a pair of multiobjective fractional variational symmetric dual problems over cones and established duality theorems. Weak, strong and converse duality theorems are established under the generalized $F$-convexity assumptions. In this paper, we introduce a pair of symmetric duals for nondifferentiable multiobjective fractional variational problems with cone constraints over arbitrary cones. On the basis of weak efficiency, we obtain symmetric duality relations for Mond-Weir-type problems under invexity and pseudo-invexity assumptions. Our duality results extend the results in Mishra et al. [16] to the cone constraints over arbitrary cones with weak efficiency.

\section{Preliminaries and notations}

The following convention for vectors $x$ and $y$ in $\mathbb{R}^{n}$ will be used:

$$
\begin{aligned}
& x>y \quad \Longleftrightarrow \quad x_{i}>y_{i} \quad \text { for all } i=1, \ldots, n, \\
& x \geqq y \quad \Longleftrightarrow \quad x_{i} \geqq y_{i} \quad \text { for all } i=1, \ldots, n, \\
& x \geq y \quad \Longleftrightarrow \quad x_{i} \geqq y_{i} \quad \text { for all } i=1, \ldots, n, \text { but } x \neq y, \\
& x \ngtr y \quad \text { is the negation of } x>y .
\end{aligned}
$$

Throughout this paper, we will use the following notations.

Let $I=[a, b]$ be a real interval, let $f:=\left(f_{1}, \ldots, f_{k}\right): I \times \mathbb{R}^{n} \times \mathbb{R}^{n} \rightarrow \mathbb{R}^{k}, g:=\left(g_{1}, \ldots, g_{k}\right): I \times$ $\mathbb{R}^{n} \times \mathbb{R}^{n} \rightarrow \mathbb{R}^{k}$ be continuously differentiable functions. In order to consider $f(t, x(t), \dot{x}(t))$, where $x: I \rightarrow \mathbb{R}^{n}$ is differentiable with derivative $\dot{x}$, denote the partial derivatives of $f$ by

$$
f_{i x}=\left[\frac{\partial f_{i}}{\partial x_{1}}, \ldots, \frac{\partial f_{i}}{\partial x_{n}}\right], \quad f_{i \dot{x}}=\left[\frac{\partial f_{i}}{\partial \dot{x}_{1}}, \ldots, \frac{\partial f_{i}}{\partial \dot{x}_{n}}\right], \quad i=1, \ldots, k
$$

Let $C\left(I, \mathbb{R}^{m}\right)$ denote the space of continuous functions $\phi: I \rightarrow \mathbb{R}^{m}$, with the uniform norm; $C_{+}\left(I, \mathbb{R}^{m}\right)$ is the cone of nonnegative functions in $C\left(I, \mathbb{R}^{m}\right)$. Denote by $X$ the space of piecewise smooth functions $x: I \rightarrow \mathbb{R}^{n}$, with the norm $\|x\|=\|x\|_{\infty}+\|D x\|_{\infty}$, where the differentiation operator $D$ is given by

$$
u=D x \quad \Longleftrightarrow x(t)=\alpha+\int_{a}^{t} u(s) d s
$$

where $\alpha$ is a given boundary value: thus $D=d / d t$ except at discontinuities. For each $t \in I$, let $B_{i}(t)$ be a positive semidefinite $n \times n$ matrix with $B_{i}(\cdot)$ continuous on $I, i=1,2, \ldots, p$ and the symbol $T$ denotes the transposition. 
Consider the following multiobjective fractional variational problem:

$(\mathrm{FVP})$

$$
\begin{aligned}
\text { Minimize } & \frac{\int_{a}^{b} f(t, x(t), \dot{x}(t)) d t}{\int_{a}^{b} g(t, x(t), \dot{x}(t)) d t} \\
& =\left(\frac{\int_{a}^{b} f_{1}(t, x(t), \dot{x}(t)) d t}{\int_{a}^{b} g_{1}(t, x(t), \dot{x}(t)) d t}, \ldots, \frac{\int_{a}^{b} f_{k}(t, x(t), \dot{x}(t)) d t}{\int_{a}^{b} g_{k}(t, x(t), \dot{x}(t)) d t}\right) \\
\text { subject to } \quad & x(a)=\alpha, \quad x(b)=\beta, \\
& h(t, x(t), \dot{x}(t)) \leqq 0, \quad t \in I,
\end{aligned}
$$

where $h: I \times \mathbb{R}^{n} \times \mathbb{R}^{n} \rightarrow \mathbb{R}^{l}$.

Assume that $g_{i}(t, x(t), \dot{x}(t))>0$ and $f_{i}(t, x(t), \dot{x}(t)) \geqq 0$ for all $i=1,2, \ldots, k$. Let $X$ denote the set of all feasible solutions of (FVP).

Definition 2.1 (1) A point $x^{*} \in X$ is said to be an efficient (Pareto optimal) solution of (FVP) if there exists no other feasible point $x \in X$ such that

$$
\frac{\int_{a}^{b} f(t, x(t), \dot{x}(t)) d t}{\int_{a}^{b} g(t, x(t), \dot{x}(t)) d t} \leq \frac{\int_{a}^{b} f\left(t, x^{*}(t), \dot{x}^{*}(t)\right) d t}{\int_{a}^{b} g\left(t, x^{*}(t), \dot{x}^{*}(t)\right) d t} .
$$

(2) A point $x^{*} \in X$ is said to be a properly efficient solution of (FVP) if it is efficient for (FVP) and if there exists a scalar $M>0$ such that, for all $i \in\{1,2, \ldots, k\}$,

$$
\begin{aligned}
& \frac{\int_{a}^{b} f_{i}\left(t, x^{*}(t), \dot{x}^{*}(t)\right) d t}{\int_{a}^{b} g_{i}\left(t, x^{*}(t), \dot{x}^{*}(t)\right) d t}-\frac{\int_{a}^{b} f_{i}(t, x(t), \dot{x}(t)) d t}{\int_{a}^{b} g_{i}(t, x(t), \dot{x}(t)) d t} \\
& \quad \leqq M\left(\frac{\int_{a}^{b} f_{j}(t, x(t), \dot{x}(t)) d t}{\int_{a}^{b} g_{j}(t, x(t), \dot{x}(t)) d t}-\frac{\int_{a}^{b} f_{j}\left(t, x^{*}(t), \dot{x}^{*}(t)\right) d t}{\int_{a}^{b} g_{j}\left(t, x^{*}(t), \dot{x}^{*}(t)\right) d t}\right)
\end{aligned}
$$

for some $j \neq i$ such that

$$
\frac{\int_{a}^{b} f_{j}(t, x(t), \dot{x}(t)) d t}{\int_{a}^{b} g_{j}(t, x(t), \dot{x}(t)) d t}>\frac{\int_{a}^{b} f_{j}\left(t, x^{*}(t), \dot{x}^{*}(t)\right) d t}{\int_{a}^{b} g_{j}\left(t, x^{*}(t), \dot{x}^{*}(t)\right) d t}
$$

whenever $x \in X$ and

$$
\frac{\int_{a}^{b} f_{i}(t, x(t), \dot{x}(t)) d t}{\int_{a}^{b} g_{i}(t, x(t), \dot{x}(t)) d t}<\frac{\int_{a}^{b} f_{i}\left(t, x^{*}(t), \dot{x}^{*}(t)\right) d t}{\int_{a}^{b} g_{i}\left(t, x^{*}(t), \dot{x}^{*}(t)\right) d t}
$$

(3) A point $x^{*} \in X$ is said to be a weakly efficient solution of (FVP) if there exists no other feasible point $x \in X$ such that

$$
\frac{\int_{a}^{b} f(t, x(t), \dot{x}(t)) d t}{\int_{a}^{b} g(t, x(t), \dot{x}(t)) d t}<\frac{\int_{a}^{b} f\left(t, x^{*}(t), \dot{x}^{*}(t)\right) d t}{\int_{a}^{b} g\left(t, x^{*}(t), \dot{x}^{*}(t)\right) d t} .
$$


Now we recall the invexity for continuous case as follows.

Definition 2.2 The vector of functionals $\int_{a}^{b} f=\left(\int_{a}^{b} f_{1}, \ldots, \int_{a}^{b} f_{k}\right)$ is said to be invex in $x$ and $\dot{x}$ if for each $y:[a, b] \rightarrow \mathbb{R}^{m}$, with $\dot{y}$ piecewise smooth, there exists a function $\eta:[a, b] \times$ $\mathbb{R}^{n} \times \mathbb{R}^{n} \times \mathbb{R}^{n} \times \mathbb{R}^{n} \rightarrow \mathbb{R}^{n}$ such that $\forall i=1,2, \ldots, k$

$$
\begin{aligned}
& \int_{a}^{b}\left\{f_{i}(t, x, \dot{x}, y, \dot{y})-f_{i}(t, u, \dot{u}, y, \dot{y})\right\} d t \\
& \quad \geqq \int_{a}^{b} \eta(t, x, \dot{x}, u, \dot{u})^{T}\left[f_{i x}(t, u, \dot{u}, y, \dot{y})-\frac{d}{d t} f_{i \dot{x}}(t, u, \dot{u}, y, \dot{y})\right] d t
\end{aligned}
$$

for all $x:[a, b] \rightarrow \mathbb{R}^{n}, u:[a, b] \rightarrow \mathbb{R}^{n}$, where $(\dot{x}(t), \dot{u}(t))$ is piecewise smooth on $[a, b]$.

Definition 2.3 The vector of functionals $-\int_{a}^{b} f=\left(-\int_{a}^{b} f_{1}, \ldots,-\int_{a}^{b} f_{k}\right)$ is said to be invex in $y$ and $\dot{y}$ if for each $x:[a, b] \rightarrow \mathbb{R}^{n}$, with $\dot{x}$ piecewise smooth, there exists function $\xi$ : $[a, b] \times \mathbb{R}^{m} \times \mathbb{R}^{m} \times \mathbb{R}^{m} \times \mathbb{R}^{m} \times \rightarrow \mathbb{R}^{m}$ such that $\forall i=1,2, \ldots, k$,

$$
\begin{aligned}
& -\int_{a}^{b}\left\{f_{i}(t, x, \dot{x}, v, \dot{v})-f_{i}(t, x, \dot{x}, y, \dot{y})\right\} d t \\
& \quad \geqq-\int_{a}^{b} \xi(t, v, \dot{v}, y, \dot{y})^{T}\left[f_{i y}(t, x, \dot{x}, y, \dot{y})-\frac{d}{d t} f_{i \dot{y}}(t, x, \dot{x}, y, \dot{y})\right] d t
\end{aligned}
$$

for all $v:[a, b] \rightarrow \mathbb{R}^{m}, y:[a, b] \rightarrow \mathbb{R}^{m}$, where $(\dot{v}(t), \dot{y}(t))$ is piecewise smooth on $[a, b]$.

Definition 2.4 The vector of functionals $\int_{a}^{b} f=\left(\int_{a}^{b} f_{1}, \ldots, \int_{a}^{b} f_{k}\right)$ is said to be pseudo-invex in $x$ and $\dot{x}$ if for each $y:[a, b] \rightarrow \mathbb{R}^{m}$, with $\dot{y}$ piecewise smooth, there exists a function $\eta:[a, b] \times \mathbb{R}^{n} \times \mathbb{R}^{n} \times \mathbb{R}^{n} \times \mathbb{R}^{n} \rightarrow \mathbb{R}^{n}$ such that $\forall i=1,2, \ldots, k$,

$$
\begin{gathered}
\int_{a}^{b} \eta(t, x, \dot{x}, u, \dot{u})^{T}\left[f_{i x}(t, u, \dot{u}, y, \dot{y})-\frac{d}{d t} f_{i \dot{x}}(t, u, \dot{u}, y, \dot{y})\right] d t \geqq 0 \\
\Rightarrow \quad \int_{a}^{b}\left\{f_{i}(t, x, \dot{x}, y, \dot{y})-f_{i}(t, u, \dot{u}, y, \dot{y})\right\} d t \geqq 0
\end{gathered}
$$

for all $x:[a, b] \rightarrow \mathbb{R}^{n}, u:[a, b] \rightarrow \mathbb{R}^{n}$, where $(\dot{x}(t), \dot{u}(t))$ is piecewise smooth on $[a, b]$.

Definition 2.5 The vector of functionals $-\int_{a}^{b} f$ is said to be pseudo-invex in $y$ and $\dot{y}$ if for each $x:[a, b] \rightarrow \mathbb{R}^{n}$, with $\dot{x}$ piecewise smooth, there exists a function $\xi:[a, b] \times \mathbb{R}^{m} \times$ $\mathbb{R}^{m} \times \mathbb{R}^{m} \times \mathbb{R}^{m} \rightarrow \mathbb{R}^{m}$ such that $\forall i=1,2, \ldots, k$,

$$
\begin{gathered}
-\int_{a}^{b} \xi(t, v, \dot{v}, y, \dot{y})^{T}\left[f_{i y}(t, x, \dot{x}, y, \dot{y})-\frac{d}{d t} f_{i \dot{y}}(t, x, \dot{x}, y, \dot{y})\right] d t \geqq 0 \\
\Rightarrow \quad-\int_{a}^{b}\left\{f_{i}(t, x, \dot{x}, v, \dot{v})-f_{i}(t, x, \dot{x}, y, \dot{y})\right\} d t \geqq 0
\end{gathered}
$$

for all $v:[a, b] \rightarrow \mathbb{R}^{m}, y:[a, b] \rightarrow \mathbb{R}^{m}$, where $(\dot{v}(t), \dot{y}(t))$ is piecewise smooth on $[a, b]$.

We consider the problem of finding functions $x:[a, b] \rightarrow \mathbb{R}^{n}$ and $y:[a, b] \rightarrow \mathbb{R}^{m}$, where $(\dot{x}(t), \dot{y}(t))$ is piecewise smooth on $[a, b]$, to solve the following pair symmetric dual prob- 
lems for nondifferentiable multiobjective fractional variational problems as follows.

$(\mathrm{NFVP})$

Minimize $\frac{\int_{a}^{b}\left\{f(t, x(t), \dot{x}(t), y(t), \dot{y}(t))+s(x(t) \mid C)-y(t)^{T} z(t)\right\} d t}{\int_{a}^{b}\left\{g(t, x(t), \dot{x}(t), y(t), \dot{y}(t))-s(x(t) \mid E)+y(t)^{T} r(t)\right\} d t}$

$$
\begin{aligned}
= & \left(\frac{\int_{a}^{b}\left\{f_{1}(t, x(t), \dot{x}(t), y(t), \dot{y}(t))+s\left(x(t) \mid C_{1}\right)-y(t)^{T} z_{1}(t)\right\} d t}{\int_{a}^{b}\left\{g_{1}(t, x(t), \dot{x}(t), y(t))-s\left(x(t) \mid E_{1}\right)+y(t)^{T} r_{1}(t)\right\} d t}, \ldots,\right. \\
& \left.\frac{\int_{a}^{b}\left\{f_{k}(t, x(t), \dot{x}(t), y(t), \dot{y}(t))+s\left(x(t) \mid C_{k}\right)-y(t)^{T} z_{k}(t)\right\} d t}{\int_{a}^{b}\left\{g_{k}(t, x(t), \dot{x}(t), y(t), \dot{y}(t))-s\left(x(t) \mid E_{k}\right)+y(t)^{T} r_{k}(t)\right\} d t}\right)
\end{aligned}
$$

subject to $x(a)=0=x(b), \quad y(a)=0=y(b)$,

$$
\begin{aligned}
& \dot{x}(a)=0=\dot{x}(b), \quad \dot{y}(a)=0=\dot{y}(b), \\
& -\sum_{i=1}^{k} \tau_{i}\left\{\left[f_{i y}-D f_{i \dot{y}}-z_{i}\right] G_{i}(x, y)-\left[g_{i y}-D g_{i \dot{y}}+r_{i}\right] F_{i}(x, y)\right\} \in C_{2}^{*}, \\
& \int_{a}^{b} y(t)^{T} \sum_{i=1}^{k} \tau_{i}\left\{\left[f_{i y}-D f_{i \dot{y}}-z_{i}\right] G_{i}(x, y)-\left[g_{i y}-D g_{i j}+r_{i}\right] F_{i}(x, y)\right\} d t \\
& \geqq 0, \\
& \tau>0, \quad \tau^{T} e=1, \quad x(t) \in C_{1}, \quad t \in I, \\
& z_{i}(t) \in D_{i}, \quad r_{i}(t) \in H_{i}, \quad i=1,2, \ldots, k .
\end{aligned}
$$

(NFVD)

Maximize

$$
\begin{gathered}
\frac{\int_{a}^{b}\left\{f(t, u(t), \dot{u}(t), v(t), \dot{v}(t))-s(v(t) \mid D)+u(t)^{T} w(t)\right\} d t}{\int_{a}^{b}\left\{g(t, u(t), \dot{u}(t), v(t), \dot{v}(t))+s(v(t) \mid H)-u(t)^{T} s(t)\right\} d t} \\
=\left(\frac{\int_{a}^{b}\left\{f_{1}(t, u(t), \dot{u}(t), v(t), \dot{v}(t))-s\left(v(t) \mid D_{1}\right)+u(t)^{T} w_{1}(t)\right\} d t}{\int_{a}^{b}\left\{g_{1}(t, u(t), \dot{u}(t), v(t), \dot{v}(t))+s\left(v(t) \mid H_{1}\right)-u(t)^{T} s_{1}(t)\right\} d t}, \ldots,\right. \\
\left.\quad \frac{\int_{a}^{b}\left\{f_{k}(t, u(t), \dot{u}(t), v(t), \dot{v}(t))-s\left(v(t) \mid D_{k}\right)+u(t)^{T} w_{k}(t)\right\} d t}{\int_{a}^{b}\left\{g_{k}(t, u(t), \dot{u}(t), v(t), \dot{v}(t))+s\left(v(t) \mid H_{k}\right)-u(t)^{T} s_{k}(t)\right\} d t}\right)
\end{gathered}
$$

subject to $u(a)=0=u(b), \quad v(a)=0=v(b)$,

$$
\begin{aligned}
& \dot{u}(a)=0=\dot{u}(b), \quad \dot{v}(a)=0=\dot{v}(b), \\
& \sum_{i=1}^{k} \tau_{i}\left\{\left[f_{i u}-D f_{i u}+w_{i}\right] G_{i}^{*}(u, v)-\left[g_{i u}-D g_{i \dot{u}}-s_{i}\right] F_{i}^{*}(u, v)\right\} \in C_{1}^{*}, \\
& \int_{a}^{b} u(t)^{T} \sum_{i=1}^{k} \tau_{i}\left\{\left[f_{i u}-D f_{i u}+w_{i}\right] G_{i}^{*}(u, v)-\left[g_{i u}-D g_{i u}-s_{i}\right] F_{i}^{*}(u, v)\right\} d t \\
& \leqq 0, \\
& \tau>0, \quad \tau^{T} e=1, \quad v(t) \in C_{2}, \quad t \in I, \\
& w_{i}(t) \in C_{i}, \quad s_{i}(t) \in E_{i}, \quad i=1,2, \ldots, k,
\end{aligned}
$$


where $f_{i}: I \times \mathbb{R}^{n} \times \mathbb{R}^{n} \times \mathbb{R}^{m} \times \mathbb{R}^{m} \rightarrow \mathbb{R}_{+}$and $g_{i}: I \times \mathbb{R}^{n} \times \mathbb{R}^{n} \times \mathbb{R}^{m} \times \mathbb{R}^{m} \rightarrow \mathbb{R}_{+} \backslash\{0\}$ are continuously differentiable functions, $C_{i}, E_{i}(1 \leqq i \leqq k)$ are a compact convex set in $\mathbb{R}^{n}$ and $D_{i}, E_{i}(1 \leqq i \leqq k)$ are a compact convex set in $\mathbb{R}^{m}, C_{1}$ and $C_{2}$ are closed convex cones in $\mathbb{R}^{n}$, $\mathbb{R}^{m}$ with nonempty interiors, respectively. $C_{1}^{*}$ and $C_{2}^{*}$ are positive polar cones of $C_{1}$ and $C_{2}$, respectively, and $s\left(x \mid C_{i}\right)=\max \left\{\langle x, y\rangle \mid y \in C_{i}\right\}$. Let $h_{i}(x)=s\left(x \mid C_{i}\right), i=1, \ldots, p$. Then $h_{i}$ is a convex function and $\partial h_{i}(x)=\left\{w \in C_{i} \mid\langle w, x\rangle=s\left(x \mid C_{i}\right)\right\}$, where $\partial h_{i}$ is the subdifferential of $h_{i}$. Let

$$
\begin{aligned}
& F_{i}(x, y)=\int_{a}^{b}\left\{f_{i}(t, x, \dot{x}, y, \dot{y})+s\left(x(t) \mid C_{i}\right)-y(t)^{T} z_{i}\right\} d t ; \\
& G_{i}(x, y)=\int_{a}^{b}\left\{g_{i}(t, x, \dot{x}, y, \dot{y})-s\left(x(t) \mid E_{i}\right)+y(t)^{T} r_{i}\right\} d t ; \\
& F_{i}^{*}(u, v)=\int_{a}^{b}\left\{f_{i}(t, u, \dot{u}, v, \dot{v})-s\left(v(t) \mid D_{i}\right)+u(t)^{T} w_{i}\right\} d t ;
\end{aligned}
$$

and

$$
G_{i}^{*}(u, v)=\int_{a}^{b}\left\{g_{i}(t, u, \dot{u}, v, \dot{v})+s\left(v(t) \mid H_{i}\right)-u(t)^{T} s_{i}\right\} d t .
$$

Let $f_{x}=f_{x}(t, x(t), \dot{x}(t), y(t), \dot{y}(t)), f_{\dot{x}}=(t, x(t), \dot{x}(t), y(t), \dot{y}(t))$, etc. All the statements above for $F_{i}, G_{i}, F_{i}^{*}$ and $G_{i}^{*}$ will be assumed to hold for subsequent results. It is to be noted that

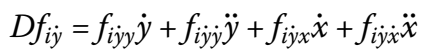

and, consequently,

$$
\begin{aligned}
\frac{\partial}{\partial y} D f_{i \dot{y}}=D f_{i \dot{y} y}, & \frac{\partial}{\partial \dot{y}} D f_{i \dot{y}}=D f_{i \ddot{y} \dot{y}}+f_{i \dot{y} y,} & \frac{\partial}{\partial \ddot{y}} D f_{i \dot{y}}=D f_{i \dot{y} \dot{y},} \\
\frac{\partial}{\partial x} D f_{i \dot{y}}=D f_{i \dot{y} x}, & \frac{\partial}{\partial \dot{x}} D f_{i \dot{y}}=D f_{i \dot{i} \dot{x}+f_{i \dot{y} x},} & \frac{\partial}{\partial \ddot{x}} D f_{i \dot{y}}=D f_{i \dot{y} \dot{x} .}
\end{aligned}
$$

In order to simplify the notations we introduce

$$
p_{i}=\frac{F_{i}(x, y)}{G_{i}(x, y)}=\frac{\int_{a}^{b}\left\{f_{i}(t, x, \dot{x}, y, \dot{y})+s\left(x(t) \mid C_{i}\right)-y(t)^{T} z_{i}\right\} d t}{\int_{a}^{b}\left\{g_{i}(t, x, \dot{x}, y, \dot{y})-s\left(x(t) \mid E_{i}\right)+y(t)^{T} r_{i}\right\} d t}, \quad i=1, \ldots, k
$$

and

$$
q_{i}=\frac{F_{i}^{*}(u, v)}{G_{i}^{*}(u, v)}=\frac{\int_{a}^{b}\left\{f_{i}(t, u, \dot{u}, v, \dot{v})-s\left(v(t) \mid D_{i}\right)+u(t)^{T} w_{i}\right\} d t}{\int_{a}^{b}\left\{g_{i}(t, u, \dot{u}, v, \dot{v})+s\left(v(t) \mid H_{i}\right)-u(t)^{T} s_{i}\right\} d t}, \quad i=1, \ldots, k
$$

and express problems (NFVP) and (NFVD) equivalently as follows.

$$
\begin{array}{lll}
(\mathrm{NFVP})^{\prime} & \text { Minimize } & p=\left(p_{1}, \ldots, p_{k}\right) \\
\text { subject to } & x(a)=0=x(b), & y(a)=0=y(b), \\
& \dot{x}(a)=0=\dot{x}(b), & \dot{y}(a)=0=\dot{y}(b),
\end{array}
$$




$$
\begin{aligned}
& \int_{a}^{b}\left\{f_{i}(t, x, \dot{x}, y, \dot{y})+s\left(x \mid C_{i}\right)-y^{T} z_{i}\right\} d t \\
& \quad-p_{i} \int_{a}^{b}\left\{g_{i}(t, x, \dot{x}, y, \dot{y})-s\left(x \mid E_{i}\right)+y^{T} r_{i}\right\} d t=0, \\
& \quad i=1, \ldots, k, \\
& -\sum_{i=1}^{k} \tau_{i}\left\{\left(f_{i y}-D f_{i j}-z_{i}\right)-p_{i}\left(g_{i y}-D g_{i \dot{y}}+r_{i}\right)\right\} \in C_{2}^{*}, \\
& \int_{a}^{b} y(t)^{T} \sum_{i=1}^{k} \tau_{i}\left\{\left(f_{i y}-D f_{i \dot{y}}-z_{i}\right)-p_{i}\left(g_{i y}-D g_{i j}+r_{i}\right)\right\} d t \\
& \geqq 0, \quad \tau^{T}, \quad x(t) \in C_{1}, \quad t \in I, \\
& \tau>0, \quad \tau^{T} e=1, \quad r_{i}(t) \in H_{i}, \quad i=1,2, \ldots, k .
\end{aligned}
$$

(NFVD)' Maximize

$q=\left(q_{1}, \ldots, q_{k}\right)$

$$
\begin{aligned}
& \text { subject to } \quad u(a)=0=u(b), \quad v(a)=0=v(b) \text {, } \\
& \dot{u}(a)=0=\dot{u}(b), \quad \dot{v}(a)=0=\dot{v}(b), \\
& \int_{a}^{b}\left\{f_{i}(t, u, \dot{u}, v, \dot{v})-s\left(v \mid D_{i}\right)+u^{T} w_{i}\right\} d t \\
& -q_{i} \int_{a}^{b}\left\{g_{i}(t, u, \dot{u}, v, \dot{v})+s\left(v \mid H_{i}\right)-u^{T} s_{i}\right\} d t=0, \\
& i=1, \ldots, k \text {, } \\
& \sum_{i=1}^{k} \tau_{i}\left\{\left(f_{i u}-D f_{i u}+w_{i}\right)-q_{i}\left(g_{i u}-D g_{i u}-s_{i}\right)\right\} \in C_{1}^{*}, \\
& \int_{a}^{b} u(t)^{T} \sum_{i=1}^{k} \tau_{i}\left\{\left(f_{i u}-D f_{i \dot{u}}+w_{i}\right)-q_{i}\left(g_{i u}-D g_{i \dot{u}}-s_{i}\right)\right\} d t \\
& \leqq 0 \\
& \tau>0, \quad \tau^{T} e=1, \quad v(t) \in C_{2}, \quad t \in I, \\
& w_{i}(t) \in C_{i}, \quad s_{i}(t) \in E_{i}, \quad i=1,2, \ldots, k .
\end{aligned}
$$

In the problems (NFVP) $)^{\prime}$ and (NFVD) ${ }^{\prime}$ above, it is to be noted that $p$ and $q$ are also nonnegative.

\section{Duality theorems}

In this section, we state duality theorems for problems (NFVP) ${ }^{\prime}$ and (NFVD)' ${ }^{\prime}$, which lead to corresponding relations between (NFVP) and (NFVD). We establish weak, strong and converse duality relations between (NFVP)' and (NFVD)'.

Theorem 3.1 (Weak duality) Let $(x(t), y(t), p, \tau, z(t), r(t))$ be feasible for $(N F V P)^{\prime}$, and let $(u(t), v(t), q, \tau, w(t), s(t))$ be feasible for $(N F V D)^{\prime}$. Assume that $\sum_{i=1}^{k} \tau_{i} \int_{a}^{b}\left\{\left(f_{i}+(\cdot)^{T} w_{i}\right)-q_{i}\left(g_{i}-\right.\right.$ 
$\left.\left.(\cdot)^{T} s_{i}\right)\right\} d t$ is pseudo-invex in $x$ and $\dot{x}$ with respect to $\eta(x, u)$ and $-\sum_{i=1}^{k} \tau_{i} \int_{a}^{b}\left\{\left(f_{i}-(\cdot)^{T} z_{i}\right)-\right.$ $\left.p_{i}\left(g_{i}+(\cdot)^{T} r_{i}\right)\right\} d t$ is pseudo-invex in $y$ and $\dot{y}$ with respect to $\xi(v, y)$, with $\eta(x, u)+u \in C_{1}$ and $\xi(v, y)+y \in C_{2} \forall t \in I$, except possibly at corners of $(\dot{x}, \dot{y})$ or $(\dot{u}, \dot{v})$. Then $p \nless q$.

Proof From (11) and $\eta(x, u)+u \in C_{1}$, we get

$$
(\eta(x, u)+u)^{T} \sum_{i=1}^{k} \tau_{i}\left\{\left(f_{i u}-D f_{i u}+w_{i}\right)-q_{i}\left(g_{i u}-D g_{i u}-s_{i}\right)\right\} d t \geqq 0 .
$$

From (12),

$$
\int_{a}^{b} \eta(x, u) \sum_{i=1}^{k} \tau_{i}\left\{\left(f_{i u}-D f_{i u}+w_{i}\right)-q_{i}\left(g_{i u}-D g_{i u}-s_{i}\right)\right\} d t \geqq 0 .
$$

Since $\sum_{i=1}^{k} \tau_{i} \int_{a}^{b}\left\{\left(f_{i}+(\cdot)^{T} w_{i}\right)-q_{i}\left(g_{i}-(\cdot)^{T} s_{i}\right)\right\} d t$ is pseudo-invex with respect to $\eta(x, u)$, it follows that

$$
\begin{aligned}
& \sum_{i=1}^{k} \tau_{i} \int_{a}^{b}\left[\left\{f_{i}(t, x, \dot{x}, v, \dot{v})+x^{T} w_{i}\right\}-q_{i}\left\{g_{i}(t, x, \dot{x}, v, \dot{v})-x^{T} s_{i}\right\}\right] d t \\
& \quad \geqq \sum_{i=1}^{k} \tau_{i} \int_{a}^{b}\left[\left\{f_{i}(t, u, \dot{u}, v, \dot{v})+u^{T} w_{i}\right\}-q_{i}\left\{g_{i}(t, u, \dot{u}, v, \dot{v})-u^{T} s_{i}\right\}\right] d t .
\end{aligned}
$$

Since $x^{T} s_{i} \leq s\left(x \mid E_{i}\right), s_{i} \in E_{i}$, and $x^{T} w_{i} \leq s\left(x \mid C_{i}\right), w_{i} \in C_{i}$, (15) can be written as

$$
\begin{aligned}
& \sum_{i=1}^{k} \tau_{i} \int_{a}^{b}\left[\left\{f_{i}(t, x, \dot{x}, v, \dot{v})+s\left(x \mid C_{i}\right)\right\}-q_{i}\left\{g_{i}(t, x, \dot{x}, v, \dot{v})-s\left(x \mid E_{i}\right)\right\}\right] d t \\
& \quad \geqq \sum_{i=1}^{k} \tau_{i} \int_{a}^{b}\left[\left\{f_{i}(t, u, \dot{u}, v, \dot{v})+u^{T} w_{i}\right\}-q_{i}\left\{g_{i}(t, u, \dot{u}, v, \dot{v})-u^{T} s_{i}\right\}\right] d t .
\end{aligned}
$$

From (4) and $\xi(v, y)+y \in C_{2}$, we get

$$
-(\xi(x, u)+y)^{T} \sum_{i=1}^{k} \tau_{i}\left\{\left(f_{i y}-D f_{i j}-z_{i}\right)-p_{i}\left(g_{i y}-D g_{i \dot{y}}+r_{i}\right)\right\} d t \geqq 0 .
$$

From (5),

$$
-\int_{a}^{b} \xi(x, u)^{T} \sum_{i=1}^{k} \tau_{i}\left\{\left(f_{i y}-D f_{i j}-z_{i}\right)-p_{i}\left(g_{i y}-D g_{i j}+r_{i}\right)\right\} d t \geqq 0 .
$$

By pseudo-invexity of $-\sum_{i=1}^{k} \tau_{i} \int_{a}^{b}\left\{\left(f_{i}-(\cdot)^{T} z_{i}\right)-p_{i}\left(g_{i}+(\cdot)^{T} r_{i}\right)\right\} d t$ with respect to $\xi(\nu, y)$, we get

$$
\begin{aligned}
& \sum_{i=1}^{k} \tau_{i} \int_{a}^{b}\left[\left\{f_{i}(t, x, \dot{x}, v, \dot{v})-v^{T} z_{i}\right\}-p_{i}\left\{g_{i}(t, x, \dot{x}, v, \dot{v})+v^{T} r_{i}\right\}\right] d t \\
& \quad \leqq \sum_{i=1}^{k} \tau_{i} \int_{a}^{b}\left[\left\{f_{i}(t, x, \dot{x}, y, \dot{y})-y^{T} z_{i}\right\}-p_{i}\left\{g_{i}(t, x, \dot{x}, y, \dot{y})+y^{T} r_{i}\right\}\right] d t
\end{aligned}
$$


Since $v^{T} r_{i} \leq s\left(v \mid H_{i}\right), r_{i} \in H_{i}$, and $v^{T} z_{i} \leq s\left(v \mid D_{i}\right), z_{i} \in D_{i}$,

$$
\begin{gathered}
-\sum_{i=1}^{k} \tau_{i} \int_{a}^{b}\left[\left\{f_{i}(t, x, \dot{x}, v, \dot{v})-s\left(v \mid D_{i}\right)\right\}-p_{i}\left\{g_{i}(t, x, \dot{x}, v, \dot{v})+s\left(v \mid H_{i}\right)\right\}\right] d t \\
\geqq-\sum_{i=1}^{k} \tau_{i} \int_{a}^{b}\left[\left\{f_{i}(t, x, \dot{x}, y, \dot{y})-y^{T} z_{i}\right\}-p_{i}\left\{g_{i}(t, x, \dot{x}, y, \dot{y})+y^{T} r_{i}\right\}\right] d t .
\end{gathered}
$$

From (16) and (17), we get

$$
\begin{aligned}
& \sum_{i=1}^{k} \tau_{i}\left(p_{i}-q_{i}\right) \int_{a}^{b} g_{i}(t, x, \dot{x}, v, \dot{v}) d t \\
& \geqq \sum_{i=1}^{k} \tau_{i}\left[\int_{a}^{b}\left\{f_{i}(t, u, \dot{u}, v, \dot{v})-s\left(v \mid D_{i}\right)+u^{T} w_{i}\right\} d t\right. \\
& \left.\quad-q_{i} \int_{a}^{b}\left\{g_{i}(t, u, \dot{u}, v, \dot{v})+s\left(v \mid H_{i}\right)-u^{T} s_{i}\right\} d t\right] \\
& \quad-\sum_{i=1}^{k} \tau_{i}\left[\int_{a}^{b}\left\{f_{i}(t, x, \dot{x}, y, \dot{y})+s\left(x \mid C_{i}\right)-y^{T} z_{i}\right\} d t\right. \\
& \left.\quad-p_{i} \int_{a}^{b}\left\{g_{i}(t, x, \dot{x}, y, \dot{y})-s\left(x \mid E_{i}\right)+y^{T} r_{i}\right\} d t\right] .
\end{aligned}
$$

From (3) and (10), (18) yields

$$
\sum_{i=1}^{k} \tau_{i}\left(p_{i}-q_{i}\right) \int_{a}^{b} g_{i}(t, x, \dot{x}, v, \dot{v}) d t \geqq 0 .
$$

Suppose, if possible, that $p_{i}<q_{i}$ for all $i$, then from $\tau \geqq 0, \tau^{T} e=1$ and $\int_{a}^{b} g_{i}(t, x, \dot{x}, v, \dot{v}) d t>$ $0, i=1,2, \ldots, k$, we have

$$
\sum_{i=1}^{k} \tau_{i}\left(p_{i}-q_{i}\right) \int_{a}^{b} g_{i}(t, x, \dot{x}, v, \dot{v}) d t<0
$$

which contradicts (19), hence $p \nless q$.

Consider the following multiobjective fractional variational problem.

$$
\begin{aligned}
(\mathrm{VP}) \text { Minimize } & \left(\int_{a}^{b}\left(f_{1}(t, x(t), \dot{x}(t))+s\left(x(t) \mid D_{1}\right)\right) d t, \ldots,\right. \\
& \left.\int_{a}^{b}\left(f_{k}(t, x(t), \dot{x}(t))+s\left(x(t) \mid D_{k}\right)\right) d t\right) \\
\text { subject to } \quad & x(a)=\alpha, \quad x(b)=\beta, \\
& g_{j}(t, x(t), \dot{x}(t)) \leqq 0, \quad j=1, \ldots, m, \\
& h_{l}(t, x(t), \dot{x}(t))=0, \quad l=1, \ldots, p,
\end{aligned}
$$


where $g_{j}: I \times \mathbb{R}^{n} \times \mathbb{R}^{n} \rightarrow \mathbb{R}^{m}$ is a continuously differentiable function, $h_{l}: I \times \mathbb{R}^{n} \times$ $\mathbb{R}^{n} \rightarrow \mathbb{R}^{p}$ is a continuously differentiable function. Let $A=\{x \in X \mid x(a)=\alpha, x(b)=$ $\left.\beta, g_{j}(t, x(t), \dot{x}(t)) \leqq 0, j=1, \ldots, m, h_{l}(t, x(t), \dot{x}(t))=0, l=1, \ldots, p\right\}$.

We need the following Fritz John necessary optimality condition in order to establish a strong duality theorem. Using the proof of Theorem 1 in [18], we obtain the following theorem.

$$
\begin{array}{lll}
(\mathrm{VP})^{\prime} & \text { Minimize } & F(x)+J(x):=\left(F_{1}(x)+J_{1}(x), \ldots, F_{k}(x)+J_{k}(x)\right) \\
\text { subject to } & G(x) \in C_{+}\left(I, \mathbb{R}^{m}\right), \\
& H(x)=0,
\end{array}
$$

where $F_{i}: X \rightarrow \mathbb{R}$ are functions defined on $x \in X, F_{i}(x)=\int_{a}^{b} f_{i}(t, x(t), \dot{x}(t)) d t, J_{i}: X \rightarrow \mathbb{R}$ are functions defined by $J_{i}(x)=\int_{a}^{b} s\left(x(t) \mid D_{i}\right) d t, G: X \rightarrow C\left(I, \mathbb{R}^{m}\right)$ are functions defined by $G(x)(t)=\left(g_{1}(t, x(t), \dot{x}(t)), \ldots, g_{m}(t, x(t), \dot{x}(x))\right)$ and $H: X \rightarrow C\left(I, \mathbb{R}^{p}\right)$ are functions defined by $H(x)(t)=\left(h_{1}(t, x(t), \dot{x}(t)), \ldots, h_{p}(t, x(t), \dot{x}(t))\right)$ and $C_{+}\left(I, \mathbb{R}^{m}\right)$. Let $S=\{x \in X \mid G(x) \in$ $\left.C_{+}\left(I, \mathbb{R}^{m}\right), H(x)=0\right\}$.

Theorem 3.2 Let $\bar{x} \in A$ be a weakly efficient solution of (VP). Suppose that there exists an $\hat{x} \in X$ such that $G(\bar{x})+G^{\prime}(\bar{x})(\hat{x}-\bar{x}) \in-\int C_{+}\left(I, \mathbb{R}^{m}\right), H^{\prime}(\bar{x})(\hat{x}-\bar{x})=0_{C\left(I, \mathbb{R}^{p}\right.}$, and the map $H^{\prime}(\bar{x})$ is surjective. Then there exist $\tau_{i} \geqq 0,\left(\tau_{1}, \ldots, \tau_{k}\right) \neq 0$ and piecewise smooth $\lambda: I \rightarrow \mathbb{R}_{+}^{m}$, $\mu: I \rightarrow \mathbb{R}^{p}$, and $w_{i} \in D_{i}, i=1, \ldots, k$, satisfying

$$
\begin{aligned}
& \sum_{i=1}^{k} \tau_{i}\left[f_{i x}(t, \bar{x}(t), \dot{\bar{x}}(t))+w_{i}\right]+\sum_{j=1}^{m} \lambda_{j}(t) g_{j x}(t, \bar{x}(t), \dot{\bar{x}}(t))+\sum_{l=1}^{p} \mu_{l}(t) h_{l x}(t, \bar{x}(t), \dot{\bar{x}}(t)) \\
& \quad=D\left[\sum_{i=1}^{k} \tau_{i} f_{i \dot{x}}(t, \bar{x}(t), \dot{\bar{x}}(t))+\sum_{j=1}^{m} \lambda_{j}(t) g_{j \dot{x}}(t, \bar{x}(t), \dot{\bar{x}}(t))+\sum_{l=1}^{p} \mu_{l}(t) h_{l \dot{x}}(t, \bar{x}(t), \dot{\bar{x}}(t))\right], \\
& \sum_{j=1}^{m} \lambda_{j}(t) g_{j}(t, \bar{x}(t), \dot{\bar{x}}(t))=0, \\
& w_{i}^{T} x(t)=s\left(x(t) \mid D_{i}\right), \quad i=1, \ldots, k
\end{aligned}
$$

for all $t \in I$.

Theorem 3.3 (Strong duality) Let $\left(x_{0}(t), y_{0}(t), p_{0}, \tau_{0}, z_{0}(t), r_{0}(t)\right)$ be a weakly efficient solution for (NFVP)' and fix $\tau=\tau_{0}$ in (NFVD)', and define $p_{0 i}=\frac{\int_{a}^{b}\left\{f_{i}\left(t, x_{0}, \dot{x}_{0}, y_{0}, \dot{y}_{0}\right)+s\left(x_{0} \mid C_{i}\right)-y_{0}^{T} z_{0 i}\right\} d t}{\int_{a}^{b}\left\{g_{i}\left(t, x_{0}, \dot{x}_{0}, y_{0}, \dot{y}_{0}\right)-s\left(x_{0} \mid E_{i}\right)+y_{0}^{T} r_{0}\right\} d t}$, $i=1,2, \ldots, k$. Suppose that all the conditions in weak duality are fulfilled. Furthermore, assume that

(I) $p_{0 i}>0, \quad i=1, \ldots, k$,

$$
\text { (II) } \begin{aligned}
\sum_{i=1}^{k} & \tau_{0 i} \int_{a}^{b} \Psi(t)^{T}\left[\left\{\left(f_{i y y}-p_{0 i} g_{i y y}\right)-D\left(f_{i j y}-p_{0 i} g_{i y \dot{y}}\right)\right\}\right. \\
& -D\left\{\left(f_{i y \dot{y}}-D f_{i \dot{y} \dot{y}}-f_{i \dot{y} y}\right)-p_{0 i}\left(g_{i \dot{y} \dot{y}}-D g_{i \dot{y} \dot{y}}-g_{i \dot{y} y}\right)\right\} \\
+ & \left.D^{2}\left\{-\left(f_{i \dot{y} \dot{y}}-p_{0 i} g_{i \dot{y} \dot{y}}\right)\right\}\right] \Psi(t) d t \leqq 0
\end{aligned}
$$


implies that $\Psi(t)=0, \forall t \in I$, and

$$
\begin{array}{r}
(\mathrm{III}) \quad\left(\int_{a}^{b}\left\{\left(f_{1 y}-D f_{1 \dot{y}}-z_{01}\right)-p_{01}\left(g_{1 y}-D g_{1 \dot{y}}+r_{01}\right)\right\} d t, \ldots,\right. \\
\left.\int_{a}^{b}\left\{\left(f_{k y}-D f_{k \dot{y}}-z_{0 k}\right)-p_{0 k}\left(g_{k y}-D g_{k j}+r_{0 k}\right)\right\} d t\right)
\end{array}
$$

is linearly independent.

Then there exist $w_{0 i}(t) \in C_{i}, s_{0 i}(t) \in E_{i}, i=1,2, \ldots, k$ such that $\left(x_{0}(t), y_{0}(t), p_{0}, \tau_{0}, w_{0}(t)\right.$, $\left.s_{0}(t)\right)$ is weakly efficient solution of (NFVD)'.

Proof Since $\left(x_{0}(t), y_{0}(t), p_{0}, \tau_{0}, z_{0}(t), r_{0}(t)\right)$ is a weakly efficient solution of (NFVP)', by Theorem 3.2, there exist $\lambda \in \mathbb{R}^{k}, \alpha \in \mathbb{R}^{k}, \zeta \in \mathbb{R}, \mu: I \longrightarrow \mathbb{R}^{k}$, piecewise smooth $\beta(t): I \longrightarrow C_{2}$ and $\rho(t): I \longrightarrow C_{1}^{*}$ such that

$$
\begin{aligned}
& \lambda_{i}-\alpha_{i}\left(g_{i}-s_{0 i}+y_{0}^{T} r_{0 i}\right)-\left(g_{i y}-D g_{i j}+r_{0 i}\right)\left(\beta-\zeta y_{0}\right)=0, \\
& i=1,2, \ldots, k \text {, } \\
& \sum_{i=1}^{k} \alpha_{i}\left[\left\{\left(f_{i x}+w_{1 i}\right)-p_{0 i}\left(g_{i x}-s_{0 i}\right)\right\}-D\left(f_{i \dot{x}}-p_{0 i} g_{i \dot{x}}\right)\right] \\
& +\sum_{i=1}^{k} \tau_{0 i}\left[\left\{\left(f_{i y x}-p_{0 i} g_{i y x}\right)-D\left(f_{i j x}-p_{0 i} g_{i y x}\right)\right\}\right. \\
& -D\left\{\left(f_{i y \dot{x}}-D f_{i \dot{y} \dot{x}}-f_{i j y x}\right)-p_{0 i}\left(g_{i y \dot{x}}-D g_{i j \dot{x}}-g_{i j y}\right)\right\} \\
& \left.+D^{2}\left\{-\left(f_{i j \dot{x}}-p_{0 i} g_{i j \dot{x}}\right)\right\}\right]\left(\beta-\zeta y_{0}\right)-\rho=0, \\
& \sum_{i=1}^{k}\left(\alpha_{i}-\zeta \tau_{0 i}\right)\left\{\left(f_{i y}-D f_{i j}-z_{0 i}\right)-p_{0 i}\left(g_{i y}-D g_{i j}+r_{0 i}\right)\right\} \\
& +\sum_{i=1}^{k} \tau_{0 i}\left[\left\{\left(f_{i y y}-p_{0 i} g_{i y y}\right)-D\left(f_{i j y}-p_{0 i} g_{i j y}\right)\right\}\right. \\
& -D\left\{\left(f_{i y \dot{y}}-D f_{i \dot{y} \dot{y}}-f_{i \dot{y} y}\right)-p_{0 i}\left(g_{i y \dot{y}}-D g_{i \dot{y} \dot{y}}-g_{i \dot{y} y}\right)\right\} \\
& \left.+D^{2}\left\{-\left(f_{i \ddot{y} \dot{y}}-p_{0 i} g_{i \dot{i} \dot{y}}\right)\right\}\right]\left(\beta-\zeta y_{0}\right)=0, \\
& \left\{\left(f_{i y}-D f_{i \dot{y}}-z_{0 i}\right)-p_{0 i}\left(g_{i y}-D g_{i \dot{y}}+r_{0 i}\right)\right\}\left(\beta-\zeta y_{0}\right)-\mu_{i}=0 \text {, } \\
& i=1, \ldots, k \text {, } \\
& \alpha_{i} p_{0 i} y_{0}+\left(\beta-\zeta y_{0}\right) \tau_{0 i} p_{0 i} \in N_{H_{i}}\left(r_{0 i}\right), \quad i=1,2, \ldots, k, \\
& \alpha_{i} y_{0}+\left(\beta-\zeta y_{0}\right) \tau_{0 i} \in N_{D_{i}}\left(z_{0 i}\right), \quad i=1,2, \ldots, k, \\
& \sum_{i=1}^{k} \alpha_{i}\left[\int_{a}^{b}\left\{\left(f_{i}+s\left(x_{0} \mid C_{i}\right)-y_{0}^{T} z_{0 i}\right)-p_{0 i}\left(g_{i}-s\left(x_{0} \mid E_{i}\right)+y_{0}^{T} r_{0 i}\right)\right\} d t\right]=0, \\
& \beta^{T} \sum_{i=1}^{k} \tau_{0 i}\left\{\left(f_{i y}-D f_{i \dot{y}}-z_{0 i}\right)-p_{0 i}\left(g_{i y}-D g_{i j}+r_{0 i}\right)\right\}=0,
\end{aligned}
$$




$$
\begin{aligned}
& \zeta y_{0}^{T} \sum_{i=1}^{k} \tau_{0 i} \int_{a}^{b}\left\{\left(f_{i y}-D f_{i j}-z_{0 i}\right)-p_{0 i}\left(g_{i y}-D g_{i j}+r_{0 i}\right)\right\} d t=0, \\
& \mu^{T} \tau_{0}=0, \\
& \rho^{T} x_{0}=0, \\
& w_{1 i} \in C_{i}, \quad w_{1 i}^{T} x_{0}=s\left(x_{0} \mid C_{i}\right), \quad i=1,2, \ldots, k \\
& s_{0 i} \in E_{i}, \quad s_{0 i}^{T} x_{0}=s\left(x_{0} \mid E_{i}\right), \quad i=1,2, \ldots, k \\
& (\lambda, \alpha, \beta(t), \zeta, \mu, \rho(t)) \geqq 0, \\
& (\lambda, \alpha, \beta(t), \zeta, \mu, \rho(t)) \neq 0 .
\end{aligned}
$$

Multiplying (22) by $\left(\beta-\zeta y_{0}\right)^{T}$,

$$
\begin{aligned}
& \sum_{i=1}^{k}\left(\alpha_{i}-\zeta \tau_{0 i}\right)\left\{\left(f_{i y}-D f_{i \dot{y}}-z_{0 i}\right)-p_{0 i}\left(g_{i y}-D g_{i \dot{y}}+r_{0 i}\right)\right\}\left(\beta-\zeta y_{0}\right) \\
& \quad+\left(\beta-\zeta y_{0}\right)^{T} \sum_{i=1}^{k} \tau_{0 i}\left[\left\{\left(f_{i y y}-p_{0 i} g_{i y y}\right)-D\left(f_{i j y}-p_{0 i} g_{i j y}\right)\right\}\right. \\
& \quad-D\left\{\left(f_{i y \dot{y}}-D f_{i \dot{y} \dot{y}}-f_{i j y}\right)-p_{0 i}\left(g_{i y \dot{y}}-D g_{i \dot{y} \dot{y}}-g_{i j y y}\right)\right\} \\
& \left.\quad+D^{2}\left\{-\left(f_{i j \dot{y}}-p_{0 i} g_{i \dot{y} \dot{y}}\right)\right\}\right]\left(\beta-\zeta y_{0}\right)=0 .
\end{aligned}
$$

Using the result in equality (23) and (29), we get

$$
\begin{aligned}
& \sum_{i=1}^{k} \alpha_{i} \mu_{i}+\left(\beta-\zeta y_{0}\right)^{T} \sum_{i=1}^{k} \tau_{0 i}\left[\left\{\left(f_{i y y}-p_{0 i} g_{i y y}\right)-D\left(f_{i j y}-p_{0 i} g_{i j y}\right)\right\}\right. \\
& \quad-D\left\{\left(f_{i y \dot{y}}-D f_{i \dot{y} \dot{y}}-f_{i \dot{y} y}\right)-p_{0 i}\left(g_{i y \dot{y}}-D g_{i \dot{y} \dot{y}}-g_{i \dot{y} y}\right)\right\} \\
& \left.\quad+D^{2}\left\{-\left(f_{i j \dot{y}}-p_{0 i} g_{i \dot{y} \dot{y}}\right)\right\}\right]\left(\beta-\zeta y_{0}\right)=0 .
\end{aligned}
$$

Since $\alpha \in \mathbb{R}_{+}^{k}, \mu \in \mathbb{R}_{+}^{k}, \alpha^{T} \mu \geqq 0$, and hence

$$
\begin{aligned}
& \sum_{i=1}^{k} \tau_{0 i} \int_{a}^{b}\left(\beta-\zeta y_{0}\right)^{T}\left[\left\{\left(f_{i y y}-p_{0 i} g_{i y y}\right)-D\left(f_{i y y}-p_{0 i} g_{i \dot{y} y}\right)\right\}\right. \\
& \quad-D\left\{\left(f_{i y \dot{y}}-D f_{i \dot{y} \dot{y}}-f_{i j y y}\right)-p_{0 i}\left(g_{i y \dot{y}}-D g_{i \dot{y} \dot{y}}-g_{i j y}\right)\right\} \\
& \left.+D^{2}\left\{-\left(f_{i \dot{y} \dot{y}}-p_{0 i} g_{i \dot{i} \dot{y}}\right)\right\}\right]\left(\beta-\zeta y_{0}\right) d t \leqq 0 .
\end{aligned}
$$

Which by virtue of the hypothesis (II) yields

$$
\beta=\zeta y_{0} \quad \forall t \in I
$$

From (22) along with (35), we obtain

$$
\sum_{i=1}^{k}\left(\alpha_{i}-\zeta \tau_{0 i}\right) \int_{a}^{b}\left\{\left(f_{i y}-D f_{i j}-z_{0 i}\right)-p_{0 i}\left(g_{i y}-D g_{i j}+r_{0 i}\right)\right\} d t=0 .
$$


By hypothesis (III),

$$
\alpha_{i}=\zeta \tau_{0 i}, \quad i=1, \ldots, k
$$

If $\zeta=0$, then (36) implies that $\alpha=0$ and using (35) $\beta=0$. From (20), we get $\lambda=0$, and from (21) $\rho=0$ and using (23), we get that $\mu=0$, which contradicts (34). Hence $\zeta>0$ and $\alpha>0$. Hence by (35), $y_{0} \in C_{2} \forall t \in I$. By (21), (35) and $\alpha_{i}=\zeta \tau_{0 i}, i=1, \ldots, k$,

$$
\sum_{i=1}^{k} \zeta \tau_{0 i}\left[\left\{\left(f_{i x}+w_{1 i}\right)-p_{0 i}\left(g_{i x}-s_{0 i}\right)\right\}-D\left(f_{i \dot{x}}-p_{0 i} g_{i \dot{x}}\right)\right]=\rho \in C_{1}^{*}
$$

Since $\sum_{i=1}^{k} \tau_{0 i}\left[\left\{\left(f_{i x}+w_{1 i}\right)-p_{0 i}\left(g_{i x}-s_{0 i}\right)\right\}-D\left(f_{i \dot{x}}-p_{0 i} g_{i \dot{x}}\right)\right] \in C_{1}^{*}$. By multiplying both sides of equation (37) by $x_{0}$, hence from (30) we get,

$$
\int_{a}^{b} x_{0}^{T} \sum_{i=1}^{k} \tau_{0 i}\left[\left\{\left(f_{i x}+w_{1 i}\right)-p_{0 i}\left(g_{i x}-s_{0 i}\right)\right\}-D\left(f_{i \dot{x}}-p_{0 i} g_{i \dot{x}}\right)\right] d t=0 .
$$

Equation (26) with $\alpha>0$ implies that

$$
\begin{aligned}
& \int_{a}^{b}\left\{\left(f_{i}\left(t, x_{0}, \dot{x}_{0}, y_{0}, \dot{y}_{0}\right)+s\left(x_{0} \mid C_{i}\right)-y_{0}^{T} z_{0 i}\right)\right. \\
& \left.\quad-p_{0 i}\left(g_{i}\left(t, x_{0}, \dot{x}_{0}, y_{0}, \dot{y}_{0}\right)-s\left(x_{0} \mid E_{i}\right)+y_{0}^{T} r_{0 i}\right)\right\} d t=0 .
\end{aligned}
$$

By (25) and the fact that $\beta=\zeta y_{0}, \alpha_{i} y_{0} \in N_{D_{i}}\left(z_{0 i}\right), i=1, \ldots, k$. Since $\alpha_{i}>0$, and so $y_{0} \in$ $N_{D_{i}}\left(z_{0 i}\right)$, hence $y_{0}^{T} z_{0 i}=s\left(y_{0} \mid D_{i}\right), i=1, \ldots, k$. By (24) and the fact that $\beta=\zeta y_{0}, \alpha_{i} p_{0 i} y_{0} \in$ $N_{H_{i}}\left(r_{0 i}\right), i=1, \ldots, k$. Since $\alpha_{i}>0, p_{0 i}>0$, and so $y_{0} \in N_{H_{i}}\left(r_{0 i}\right)$, hence $y_{0}^{T} r_{0 i}=s\left(y_{0} \mid H_{i}\right), i=$ $1, \ldots, k$. Thus, from (31), (32) and $y_{0}^{T} z_{0 i}=s\left(y_{0} \mid D_{i}\right), y_{0}^{T} r_{0 i}=s\left(y_{0} \mid H_{i}\right), i=1, \ldots, k$, equation (38) implies

$$
\begin{aligned}
& \int_{a}^{b}\left\{\left(f_{i}\left(t, x_{0}, \dot{x}_{0}, y_{0}, \dot{y}_{0}\right)-s\left(y_{0} \mid D_{i}\right)+x_{0}^{T} w_{1 i}\right)\right. \\
& \left.\quad-p_{0 i}\left(g_{i}\left(t, x_{0}, \dot{x}_{0}, y_{0}, \dot{y}_{0}\right)+s\left(y_{0} \mid H_{i}\right)-x_{0}^{T} s_{0 i}\right)\right\} d t=0,
\end{aligned}
$$

and

$$
\begin{aligned}
p_{0 i} & =\frac{\int_{a}^{b}\left\{f_{i}\left(t, x_{0}, \dot{x}_{0}, y_{0}, \dot{y}_{0}\right)+s\left(x_{0} \mid C_{i}\right)-y_{0}^{T} z_{0 i}\right\} d t}{\int_{a}^{b}\left\{g_{i}\left(t, x_{0}, \dot{x}_{0}, y_{0}, \dot{y}_{0}\right)-s\left(x_{0} \mid E_{i}\right)+y_{0}^{T} r_{0 i}\right\} d t} \\
& =\frac{\int_{a}^{b}\left\{f_{i}\left(t, x_{0}, \dot{x}_{0}, y_{0}, \dot{y}_{0}\right)-s\left(y_{0} \mid D_{i}\right)+x_{0}^{T} w_{1 i}\right\} d t}{\int_{a}^{b}\left\{g_{i}\left(t, x_{0}, \dot{x}_{0}, y_{0}, \dot{y}_{0}\right)+s\left(y_{0} \mid H_{i}\right)-x_{0}^{T} s_{0 i}\right\} d t} \\
& =q_{0 i} .
\end{aligned}
$$

Thus $\left(x_{0}, y_{0}, p_{0}, \tau_{0}, z_{0}, r_{0}\right)$ is feasible for (NFVD)' , and the objective values of (NFVP)' and $(\mathrm{NFVD})^{\prime}$ are equal there. Clearly, $\left(x_{0}, y_{0}, p_{0}, \tau_{0}, z_{0}, r_{0}\right)$ is weakly efficient for (NFVD)'. If $\left(x_{0}, y_{0}, p_{0}, \tau_{0}, z_{0}, r_{0}\right)$ is not weakly efficient for $(\mathrm{NFVD})^{\prime}$, then for some feasible $(\tilde{x}, \tilde{y}, \tilde{p}$, $\tilde{\tau}, \tilde{z}, \tilde{r})$ of $(\mathrm{NFVD})^{\prime}$, there exist $w_{0 i}(t) \in C_{i}, s_{0 i}(t) \in E_{i}$ such that $p_{0}<\tilde{p}$, with $\widetilde{p}_{i}=$ 
$\frac{\int_{a}^{b}\left\{f_{i}(t, \tilde{x}, \tilde{\dot{x}}, \tilde{y}, \tilde{\hat{y}})-s\left(\tilde{y} \mid D_{i}\right)+\tilde{x}^{T} w_{0 i}\right\} d t}{\int_{a}^{b}\left\{g_{i}(t, \tilde{x}, \dot{\tilde{x}}, \tilde{y}, \dot{\tilde{y}})+s\left(\tilde{y} \mid H_{i}\right)-\tilde{x}^{T} s_{0 i}\right\} d t}, i=1, \ldots, k$. Since $g_{i}\left(t, x_{0}, \dot{x}_{0}, y_{0}, \dot{y}_{0}\right)>0, i=1, \ldots, k$, it follows that $\sum_{i=1}^{k} \tau_{i}\left(p_{0 i}-\tilde{p}_{i}\right) \int_{a}^{b} g_{i}\left(t, x_{0}, \dot{x}_{0}, y_{0}, \dot{y}_{0}\right) d t<0$, which contradicts by weak duality, equation (19). Thus $\left(x_{0}, y_{0}, p_{0}, \tau_{0}, z_{0}, r_{0}\right)$ is a weakly efficient solution of (NFVD)'. Hence the result holds.

Theorem 3.4 (Converse duality) Let $\left(x_{0}(t), y_{0}(t), q_{0}, \tau_{0}, w_{0}(t), s_{0}(t)\right)$ be a weakly efficient solution for (NFVD)' and fix $\tau=\tau_{0}$ in $(\mathrm{NFVP})^{\prime}$, and define

$$
q_{0 i}=\frac{\int_{a}^{b}\left\{f_{i}\left(t, x_{0}, \dot{x}_{0}, y_{0}, \dot{y}_{0}\right)-s\left(y_{0} \mid D_{i}\right)+x_{0}^{T} w_{0 i}\right\} d t}{\int_{a}^{b}\left\{g_{i}\left(t, x_{0}, \dot{x}_{0}, y_{0}, \dot{y}_{0}\right)+s\left(y_{0} \mid H_{i}\right)-x_{0}^{T} s_{0 i}\right\} d t}, \quad i=1,2, \ldots, k .
$$

Suppose that all the conditions in weak duality are fulfilled. Furthermore, assume that

(I) $q_{0 i}>0, \quad i=1, \ldots, k$,

(II) $\sum_{i=1}^{k} \tau_{0 i} \int_{a}^{b} \Psi(t)^{T}\left[\left\{\left(f_{i x x}-q_{0 i} g_{i x x}\right)-D\left(f_{i \dot{x} x}-q_{0 i} g_{i x \dot{x}}\right)\right\}\right.$

$$
\begin{aligned}
& -D\left\{\left(f_{i x \dot{x}}-D f_{i \dot{x} \dot{x}}-f_{i \dot{x} x}\right)-q_{0 i}\left(g_{i \dot{x} \dot{x}}-D g_{i \dot{x} \dot{x}}-g_{i \dot{x} \dot{x}}\right)\right\} \\
& \left.+D^{2}\left\{-\left(f_{i \dot{x} \dot{x}}-q_{0 i} g_{i \dot{x} \dot{x}}\right)\right\}\right] \Psi(t) d t \geqq 0
\end{aligned}
$$

implies that $\Psi(t)=0, \forall t \in I$, and

$$
\text { (III) } \begin{array}{r}
\left(\int_{a}^{b}\left\{\left(f_{1 x}-D f_{1 \dot{x}}+w_{01}\right)-q_{01}\left(g_{1 x}-D g_{1 \dot{x}}-s_{01}\right)\right\} d t, \ldots,\right. \\
\left.\int_{a}^{b}\left\{\left(f_{k x}-D f_{k \dot{x}}+w_{0 k}\right)-q_{0 k}\left(g_{k x}-D g_{k \dot{x}}-s_{0 k}\right)\right\} d t\right)
\end{array}
$$

is linearly independent.

Then there exist $z_{0 i}(t) \in D_{i}, r_{0 i}(t) \in H_{i}, i=1,2, \ldots, k$ such that $\left(x_{0}(t), y_{0}(t), q_{0}, \tau_{0}, z_{0}(t)\right.$, $\left.r_{0}(t)\right)$ is weakly efficient solution of (NFVP)'.

Proof It is analogous to the proof of the lines of Theorem 3.3.

Remark 3.1 (1) When $C=D=E=H=\{0\}$, then the support functions and inner products in the problems (NFVP) and (NFVD) in the draft disappear, and hence (NFVP) and (NFVD) in the draft collapse to (P) and (D) in the paper of Ahmad, Sharma (EJOR, Vol. 188, 2008, pp. 695-704) [19].

(2) When $C_{1}=\mathbb{R}_{+}^{n}$, and $C_{2}=\mathbb{R}_{+}^{m}$, then the problems (NFVP) and (NFVD) reduce to those considered by Mishra et al. [16], respectively.

Competing interests

The authors declare that they have no competing interests.

\section{Authors' contributions}

DSK introduced a pair of symmetric dual problems for nondifferentiable multiobjective fractional variational problems with cone constraints and established symmetric duality relations for Mond-Weir-type problems under invexity and pseudoinvexity assumptions. YMK and MHK carried out the symmetric duality studies for nondifferentiable multiobjective fractional variational problems, participated in the sequence alignment and drafted the manuscript. All authors read and approved the final manuscript. 


\section{Author details}

${ }^{1}$ Department of Applied Mathematics, Pukyong National University, Busan, 608-737, Republic of Korea. ${ }^{2}$ School of Free Major, Tongmyong University, Busan, 608-711, Korea.

\section{Acknowledgements}

This work was supported by the Research Grant of Pukyong National University (2013). The authors wish to thank the anonymous referees for their suggestions and comments.

\section{Received: 4 April 2013 Accepted: 20 August 2013 Published: 13 September 2013}

\section{References}

1. Dorn, WS: A symmetric dual theorem for quadratic programs. J. Oper. Res. Soc. Jpn. 2, 93-97 (1960)

2. Dantzig, GB, Eisenberg, E, Cottle, RW: Symmetric dual nonlinear programs. Pac. J. Math. 15, 809-812 (1965)

3. Mond, B, Hanson, MA: Symmetric duality for variational problems. J. Math. Anal. Appl. 23, 161-172 (1968)

4. Suneja, SK, Aggarwal, S, Davar, S: Multiobjective symmetric duality involving cones. Eur. J. Oper. Res. 141, 471-479 (2002)

5. Khurana, S: Symmetric duality in multiobjective programming involving generalized cone-invex functions. Eur. J. Oper. Res. 165, 592-597 (2005)

6. Kim, MH, Kim, DS: Non-differentiable symmetric duality for multiobjective programming with cone constraints. Eur. J. Oper. Res. 188, 652-661 (2008)

7. Ahmad, I, Husain, Z: Multiobjective mixed symmetric duality involving cones. Comput. Math. Appl. 59(1), 319-326 (2010)

8. Chandra, S, Craven, BD, Mond, B: Symmetric dual fractional programming. Z. Oper.-Res. 29, 59-64 (1985)

9. Mond, B, Schechter, M: Nondifferentiable symmetric duality. Bull. Aust. Math. Soc. 53, 177-188 (1996)

10. Yang, XM, Teo, KL, Yang, XQ: Symmetric duality for a class of nonlinear fractional programming problems. J. Math Anal. Appl. 271, 7-15 (2002)

11. Gulati, TR, Husain, I, Ahmed, A: Symmetric duality with invexity in static and continuous fractional programming. Optimization 40, 41-56 (1997)

12. Gulati, TR, Husain, I, Ahmed, A: Symmetric duality for multiobjective variational problems. J. Math. Anal. Appl. 210 22-38 (1997)

13. Kim, DS, Lee, WJ: Symmetric duality for multiobjective variational problems with invexity. J. Math. Anal. Appl. 218, 34-48 (1998)

14. Chen, $X$ : Symmetric duality for the multiobjective fractional variational problem with partial invexity. J. Math. Anal. Appl. 245, 105-123 (2000)

15. Kim, DS, Lee, WJ, Schaible, S: Symmetric duality for invex multiobjective fractional variational problems. J. Math. Anal. Appl. 289, 505-521 (2004)

16. Mishra, SK, Wang, SY, Lai, KK: Symmetric duality for a class of nondifferentiable multi-objective fractional variational problems. J. Math. Anal. Appl. 333, 1093-1110 (2007)

17. Ahmad, I, Sharma, S: Symmetric duality for multiobjective fractional variational problems involving cones. Eur. J. Oper Res. 188, 695-704 (2008)

18. Chandra, S, Craven, BD, Husain, I: A class of nondifferentiable continuous programming problems. J. Math. Anal. Appl. $107,122-131(1985)$

19. Ahmad, I: Symmetric duality for multiobjective fractional variational problems with generalized invexity. Inf. Sci. 176 2192-2207 (2006)

doi:10.1186/1029-242X-2013-434

Cite this article as: Kang et al.: Symmetric duality for nondifferentiable multiobjective fractional variational problems involving cones. Journal of Inequalities and Applications 2013 2013:434.

\section{Submit your manuscript to a SpringerOpen ${ }^{\ominus}$ journal and benefit from:}

- Convenient online submission

Rigorous peer review

- Immediate publication on acceptance

Open access: articles freely available online

- High visibility within the field

- Retaining the copyright to your article 